\title{
CYCLIC EXTENSIONS WITHOUT RELATIVE INTEGRAL BASES
}

\section{LEON R. MCCULLOH}

Let $K$ be an algebraic number field and $o$ the ring of algebraic integers in $K$. If $\mathfrak{o}$ is a principal ideal domain (p.i.d.) then any finite extension $\Lambda / K$ has an integral basis over $o$ (i.e., the ring of integers $\mathfrak{D}=\mathfrak{O}(\Lambda)$ of $\Lambda$ is a free $\mathfrak{D}$-module). The converse of this was shown by Mann [5]. More precisely, he proved that if 0 is not a p.i.d., there is a quadratic extension $\Lambda / K$ which has no in tegral basis over $\mathfrak{o}$. Thus $\mathrm{o}$ is a p.i.d. if and only if every quadratic extension of $K$ has an integral basis. One can also show ([7] or the corollary below) that if $K$ contains a primitive cube root of 1 , then $\mathfrak{D}$ is a p.i.d. if and only if every cyclic extension of degree 3 has an integral basis. However, the analogous theorem with 3 replaced by a prime $p>3$ is false.

The problem considered here is the following. Given a finite group $G$ of order $n$ and an algebraic number field $K$, consider all normal extensions $\Lambda / K$ with Galois group isomorphic to $G$. What are the $\mathfrak{D}$ module types of the $\mathfrak{D}(\Lambda)$ for these extensions? In particular, when are all the $\mathfrak{O}(\Lambda)$ free? In Theorems 1 and 2 we answer these questions in the case that $G$ is cyclic of order $n$ and $K$ contains the $n$th roots of unity.

A finitely generated torsion free $\mathbf{D}$-module $M$ of a given $\mathbf{D}$-rank is characterized by its Steinitz class $C(M)=C_{0}(M)$ which is an o-ideal class of $K$. Specifically, $M \cong 0^{(r-1)} \oplus J$ where $r$ is the $\mathfrak{D}$-rank of $M$, $\mathrm{D}^{(r-1)}$ is a free $\mathfrak{D}$-module of rank $r-1$, and $J$ is any ideal in the class $C(M)$. If $\Lambda / K$ is a finite extension, let $\mathfrak{D}=\mathfrak{D}(\mathfrak{D}(\Lambda) / \mathfrak{0})$ be the discriminant ideal and let $\Delta=\Delta(\Lambda / K)$ be the discriminant of a basis of $\Lambda / K$. It was shown by Artin that the ideal $(\mathfrak{D} /(\Delta))^{1 / 2}$ is an 0 -ideal lying in $C_{0}(\mathfrak{O}(\Lambda)$ ). (For proofs of the above remarks, see Artin [1] or Fröhlich [2] and [3].)

Definition. If $l$ is an odd prime, let $d(l)=(l-1) / 2$, and let $d(2)=1$. We define, for any integer $n, d(n)=$ g.c.d. $\{d(l) \mid l$ is a prime divisor of $n\}$.

Theorem 1. Let $\Lambda / K$ be normal of degree $n$. Then $C_{0}(\mathfrak{O}(\Lambda))$ is a $d(n)$ th power in the ideal class group of $\mathrm{D}$.

Proof. If $n$ is even, $d(n)=1$ and the theorem is trivial. If $n$ is odd,

Presented to the Society, April 23, 1966; received by the editors February 17, 1966. 
the discriminant $\Delta$ of any basis of $\Lambda / K$ is a square in $K$, so $C(\mathfrak{D})$ is the class of $\mathfrak{D}^{1 / 2}$. Let $\mathfrak{p}$ be any prime of $\mathfrak{D}$ and suppose $\mathfrak{p} \cdot \mathfrak{D}=\left(\mathfrak{B}_{1} \cdots \mathfrak{B}_{g}\right)^{e}$ where each prime $\mathfrak{B}_{j}$ is of degree $f$ over $\mathfrak{p}$. Let $G_{i}(i=0, \cdots, \nu)$ be the ramification groups of $\mathfrak{B}_{1}$. Then, by the Hilbert formula, $\mathfrak{D}$ is exactly divisible by $\mathfrak{p}^{r}$ where $r=f g \cdot \sum\left\{\left(\#\left(G_{i}\right)-1\right) \mid i=0, \cdots, \nu\right\}$. Clearly $2 d(n) \mid\left(\#\left(G_{i}\right)-1\right)$ for each $i$, so $d(n) \mid(r / 2)$.

TheOREM 2. Let $n$ be a positive integer and let $\zeta \in K$ where $\zeta$ is a primitive nth root of 1 . If $\mathfrak{c}$ is any $\mathrm{D}$-ideal class of $K$, there is a cyclic extension $\Lambda / K$ of degree $n$ with $C_{0}(\mathfrak{O}(\Lambda))=c^{d(n)}$. (In fact, there are infinitely many such extensions, and they may be chosen so that $\mathfrak{D}(\mathfrak{D}(\Lambda) / \mathrm{o})$ is relatively prime to any preassigned $\mathfrak{D}$-ideal $\mathfrak{b}$ of $K$.)

The following is an immediate consequence.

Corollary. (Same hypothesis.) $\mathfrak{D}(\Lambda)$ is a free $\mathfrak{0}$-module for every cyclic extension $\Lambda / K$ of degree $n$ if and only if $d(n)$ is divisible by the exponent of the ideal class group of $\mathbf{D}$.

Proof of Theorem 2. We prove the theorem first for the case $n=l^{r}$ where $l$ is a prime. If $\mathfrak{m}$ is any ideal in $\mathfrak{o}$, there are infinitely many prime ideals in any ideal class mod $\mathrm{m}$. (The ideal class group $\bmod \mathfrak{m}$ is the quotient of the group of all $\mathfrak{D}$-ideals prime to $\mathfrak{m}$ modulo the subgroup of principal ideals of form $\alpha \cdot \mathfrak{D}$ where $\alpha \equiv 1(\bmod \mathrm{m})$ ).

First suppose $l$ is odd. Let $c$ be any ideal class and let $t>1$ be any integer such that $c^{t}=c$. (We may suppose $t$ is odd.) Let $\mathfrak{p}$ be any prime ideal in $c$ such that $p \nmid l$. Choose distinct primes $p_{1}, \cdots, p_{t}$ in the same ideal class $\bmod \mathfrak{m}$ as $\mathfrak{p}$, where we take $\mathfrak{m}=(1-\zeta)^{2^{2 r}}$. Then choose primes $\mathfrak{q}_{1}, \cdots, \mathfrak{q}_{t}$ in the inverse ideal class of $\mathfrak{p} \bmod \mathfrak{m}$. Then choose positive integers $a_{1}, \cdots, a_{t}$, prime to $l$, such that $\sum a_{i}=l t$. (For example, $a_{i}=l-1$ for $1 \leqq i \leqq(t-1) / 2, a_{i}=l+1$ for $(t+1) / 2 \leqq i \leqq t-1$, and $a_{t}=l+2$.) Then $\left(\prod_{i=1}^{t} p_{i}^{a_{i}}\right) \cdot\left(\prod_{i=1}^{t} \mathfrak{q}_{i}\right)^{l}=\mu \cdot \mathfrak{o}$, a principal ideal where $\mu \equiv 1(\bmod m)$. If $\alpha$ is a root of $f(x)=X^{l^{r}}-\mu$, then $\Lambda=K(\alpha)$ is a cyclic extension of $K$ of degree $l^{r}$. We show that $C(\supseteq(\Lambda))=\mathfrak{c}^{(l-1) / 2}$. To do this, we must compute $\mathfrak{D}(\mathfrak{D}(\Lambda) / \mathfrak{D})$.

First we show that no higher (i.e., wild) ramification occurs. For let $\mathfrak{l}$ be a prime of $K$ dividing $l$, and suppose $\mathfrak{l}^{a}$ exactly divides $(1-\zeta)$. Let $\mathfrak{R}$ be a prime of $\Lambda$ dividing $\mathfrak{l}$, say $\mathfrak{R}^{b}$ exactly divides $\mathfrak{l}$. Now, $1-\mu=\prod_{\sigma}(1-\sigma(\alpha))$ where $\sigma$ runs over the Galois group $G$ of $\Lambda / K$. Since $1-\mu$ is divisible by $m=(1-\zeta)^{l^{2 r}}$, at least one of the factors (which we may take to be $(1-\alpha)$ ) is divisible at least by $\mathfrak{R}^{a b l^{r}}$. But, for any $\sigma \in G, \sigma \neq 1$, we have $\sigma(\alpha)-\alpha=\left(\zeta^{j}-1\right) \alpha$ for some $0<j<l^{r}$. Since $\ell \nmid \alpha$ and $\left(\zeta^{j}-1\right) \mid\left(\zeta^{l^{r-1}}-1\right)$ which is exactly divisible by $\left\{a b l^{r-1}\right.$, we have $\sigma(\alpha)-\alpha$ divisible at most by $\mathfrak{R a b l}^{r-1}$. Hence, in the $\mathbb{R}$-adic 
metric on $\Lambda, \alpha$ is closer to 1 than to any of its conjugates $\sigma(\alpha)$. Then, by Krasner's Lemma (see, e.g., [8, p. 82]), letting $K^{*}$ and $\Lambda^{*}$ denote the completions of $K$ and $\Lambda$ at $\mathfrak{R}$, we have $\Lambda^{*}=K^{*}(\alpha) \subseteq K^{*}(1)=K^{*}$. Hence, $\mathfrak{Z}$ is unramified over $K$ and, indeed, of degree 1 over $K$.

Since $f^{\prime}(\alpha)=l^{r}(\alpha)^{l^{r}-1}$, the only possible divisors of $\mathfrak{D}$ are the divisors of $\mu$. Clearly $\mathfrak{p}_{1}, \cdots, \mathfrak{p}_{t}$ are completely ramified in $\Lambda$ so that $\mathfrak{D}$ is exactly divisible by $p_{i}^{l^{r}-1}$. On the other hand, it is easily seen that the inertial field for any prime divisor of $q_{j}(1 \leqq j \leqq t)$ is $K\left((\mu)^{1 / l}\right)$ $=K\left(\alpha^{l^{r-1}}\right)$. (To see this: $\mathfrak{q}_{i}$ is unramified in $K\left((\mu)^{1 / l}\right)$, for we can easily find $\mu^{\prime}=\beta^{l} \mu$ where $q_{j}$ is prime to $\mu^{\prime}$ and $K\left((\mu)^{1 / l}\right)=K\left(\left(\mu^{\prime}\right)^{1 / l}\right)$. Also, clearly, the ramification index of any divisor of $\mathfrak{q}_{j}$ in $\Lambda$ is at least $l^{r-1}$, whence it is exactly $l^{r-1}$.) Thus, $\mathfrak{D}$ is exactly divisible by $q_{j}^{\left(l^{r-1}-1\right)}$ $=q_{j}^{r-l}$. Hence,

$$
\begin{aligned}
\mathfrak{D}^{1 / 2} & =\left(\prod_{i=1}^{t} \mathfrak{p}_{i}\right)^{\left(l^{r}-1\right) / 2}\left(\prod_{j=1}^{t} \mathfrak{q}_{j}\right)^{\left(l^{r}-l\right) / 2} \sim \mathfrak{p}^{-t\left(l^{r}-1\right) / 2} \mathfrak{p}^{t\left(l^{r}-l\right) / 2} \\
& =\mathfrak{p}^{t(l-1) / 2} \sim \mathfrak{p}^{(l-1) / 2} .
\end{aligned}
$$

(Here, $\sim$ means "belongs to the same ideal class as.") Hence $C(\mathfrak{D}(\Lambda))$ $=\mathrm{c}^{(l-1) / 2}$.

The case $l=2$ is similar. Choose a prime $p \nmid 2$ in the class c. Take primes $p_{1}$ and $p_{3}$ in the same class mod $m$ as $\mathfrak{p}$, and take $p_{2}$ in the class of $\mathfrak{p}^{-2} \bmod \mathfrak{m}$, where $\mathfrak{m}$ is a power of 2 large enough to avoid higher ramification. Then $p_{1} p_{2}^{2} p_{2}^{3}=(\mu)$ where $\mu \equiv 1(\bmod m)$. Let $\alpha$ be a root of $f(x)=x^{2^{r}}-\mu$ and consider $\Lambda=K(\alpha)$. Then $\mathfrak{D}=\left(p_{1} p_{3}\right)^{2^{r}-1} p_{2}^{2^{r}-2}$. Also, $f^{\prime}(\alpha)=2^{r} \cdot \alpha^{2^{r}-1}$, so if $\Delta$ is the discriminant of the basis $1, \alpha, \alpha^{2}, \cdots$, $\alpha^{2^{r}-1}$, then $(\Delta)=\left(2^{r}\right)^{2^{r}(\mu)^{2^{r}-1}}$ o. Hence $\left((\mathfrak{D} / \Delta)^{1 / 2}\right) \sim \mathfrak{p}_{3}^{-\left(2^{r}-1\right)} \mathfrak{p}_{2}^{-2^{r-1}}$ $\sim\left(\mathfrak{p}^{1-2^{r}}\right) \mathfrak{p}^{2^{r}}=\mathfrak{p}$. Hence $C(\mathfrak{O}(\Lambda))=\mathfrak{c}$. This completes the proof of Theorem 2 for the case $n=l^{r}$.

Before proving the general case, we prove the following lemma. (This is well known, but it seems to be hard to find in print. Compare $[4$, p. 202] and $[6$, p. 72]).

Lemma. Let $\Lambda_{1}$ and $\Lambda_{2}$ be linearly disjoint over $K$ (i.e. $\Lambda_{1} \cdot \Lambda_{2} \cong \Lambda_{1} \otimes{ }_{K} \Lambda_{2}$ ). Let $\mathfrak{D}_{i}=\mathfrak{D}\left(\Lambda_{i}\right)$. If $\mathfrak{D}\left(\mathfrak{O}_{1} / \mathfrak{D}\right)$ and $\mathfrak{D}\left(\mathfrak{D}_{2} / \mathfrak{D}\right)$ are relatively prime, then the maximal D-order of $\Lambda_{1} \otimes_{K} \Lambda_{2}$ is $\mathfrak{D}_{1} \otimes_{0} \mathfrak{D}_{2}$ and its discriminant over $\mathfrak{D}$ is $\mathfrak{D}\left(\mathfrak{D}_{1} / \mathfrak{p}\right)^{\left[\Lambda_{2}: K\right]} \mathfrak{D}\left(\mathfrak{D}_{2} / \mathfrak{p}\right)^{\left[\Lambda_{1}: K\right]}$.

Proof. Let $\mathfrak{D}^{\prime}$ be the maximal $\mathfrak{D}$-order of $\Lambda_{1} \otimes_{K} \Lambda_{2}$. Then $\mathfrak{D}^{\prime}$ $\supseteq \mathfrak{O}_{1} \otimes_{0} \mathfrak{D}_{2}$, and $\mathfrak{D}\left(\mathfrak{D}_{1} \otimes_{0} \mathfrak{D}_{2} / \mathfrak{D}_{2}\right)=\mathfrak{D}\left(\mathfrak{D}^{\prime} / \mathfrak{D}_{2}\right)\left[\mathfrak{D}^{\prime}: \mathfrak{D}_{1} \otimes_{0} \mathfrak{O}_{2}\right]^{2}$ where the notation $[M: N]$ denotes the module index (see Fröhlich [2], [3] and $[4])$. Also,

$$
\mathfrak{D}\left(\mathfrak{D}^{\prime} / \mathfrak{0}\right)=N_{\Lambda_{i} / K}\left(\mathfrak{I}\left(\mathfrak{D}^{\prime} / \mathfrak{D}_{i}\right)\right) \cdot \mathfrak{D}\left(\mathfrak{D}_{i} / \mathfrak{0}\right)^{\left[\Delta_{j}: K\right]}
$$


where the pair $(i, j)$ is $(1,2)$ or $(2,1)$.

Now, since $\mathfrak{D}\left(\mathfrak{D}^{\prime} / \mathfrak{D}_{i}\right)$ divides $\mathfrak{D}\left(\mathfrak{D}_{1} \otimes \mathfrak{D}_{2} / \mathfrak{D}_{i}\right)=\mathfrak{D}\left(\mathfrak{D}_{j} / \mathfrak{o}\right) \cdot \mathfrak{D}_{i}$, $N_{\Lambda_{i} / K}\left(\mathfrak{D}\left(\mathfrak{D}^{\prime} / \mathfrak{D}_{i}\right)\right)$ divides $\mathfrak{D}\left(\mathfrak{D}_{i} / \mathfrak{D}\right)^{\left[\Delta_{i} / K\right]}$ and is, therefore, prime to $\mathfrak{D}\left(\mathfrak{D}_{i} / \mathfrak{o}\right)$. Hence, from (1) we have $N_{\Lambda_{i} / K}\left(\mathfrak{D}\left(\mathfrak{D}^{\prime} / \mathfrak{D}_{i}\right)\right)=\left(\mathfrak{D}\left(\mathfrak{D}_{j} / \mathfrak{o}\right)^{\left[\Lambda_{i} / K\right]}\right.$ and $\mathfrak{D}\left(\mathfrak{D}^{\prime} / \mathfrak{D}_{i}\right)=\mathfrak{D}\left(\mathfrak{D}_{1} \otimes \mathfrak{D}_{2} / \mathfrak{D}_{i}\right)$ whence $\left[\mathfrak{D}^{\prime}: \mathfrak{D}_{1} \otimes \mathfrak{D}_{2}\right]=(1)$ and $\mathfrak{D}^{\prime}=\mathfrak{D}_{1} \otimes \mathfrak{D}_{2}$.

We next prove Theorem 2 in the general case. Let $n=\prod_{i=1}^{s} l_{i}^{r_{i}}$ where the $l_{i}$ are distinct primes. Let $d=d(n)$. For each $i$, let $d\left(l_{i}\right)$ $=d \cdot h_{i}$. Then g.c.d. $\left\{h_{i} \mid 1 \leqq i \leqq s\right\}=1$ and $\left(h_{i}, l_{i}\right)=1$. Hence g.c.d. $\left\{h_{i} n / l_{i}{ }^{r_{i}} \mid 1 \leqq i \leqq s\right\}=1$. For, suppose to the contrary that the prime $p$ is a common divisor. We may suppose $p \nmid h_{1}$ whence $p \mid\left(n / l_{1}^{r_{1}}\right)$ so $p=l_{2}$, say. But then $p \nmid\left(h_{2} n / l_{2}^{2}\right)$.

Choose integers $x_{i}$ such that $\sum\left\{x_{i} h_{i} n / l_{i}^{r_{i}} \mid 1 \leqq i \leqq s\right\}=1$. Then $d=\sum\left\{x_{i} d\left(l_{i}\right) n / l_{i}^{r_{i}} \mid 1 \leqq i \leqq s\right\}$. Choose cyclic extensions $\Lambda_{i} / K$ of degree $l_{i}^{r_{i}}$ having $C\left(\mathfrak{D}\left(\Lambda_{i}\right)\right)=\mathfrak{c}^{x_{i} d\left(l_{i}\right)}$ and such that the $\mathfrak{D}\left(\mathfrak{D}\left(\Lambda_{i}\right) / \mathfrak{o}\right)$ are relatively prime in pairs. Then $\Lambda=\Lambda_{1} \cdots \Lambda_{s} \cong \Lambda_{1} \otimes_{K} \cdots \otimes_{K} \Lambda_{s}$ and

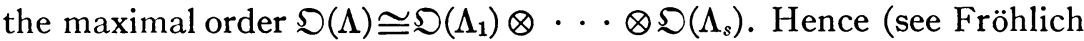
$[3$, p. 32])

$$
C(\Im(\Lambda))=\prod\left\{C\left(\Im\left(\Lambda_{i}\right)\right)^{n / l_{i}^{r_{i}}} \mid 1 \leqq i \leqq s\right\}=\mathfrak{c}^{d} .
$$

\section{REFERENCES}

1. E. Artin, Questions de base minimale dans la théorie des nombres algêbriques, Colloques Internationaux du Centre National de la Récherche Scientifique, No. XXIV, pp. 19-20, Paris, 1950.

2. A. Fröhlich, Discriminants of algebraic number fields, Math. Z. 74 (1960), $18-28$.

3. - Ideals in an extension field as modules over the algebraic integers in a finite number field, Math. Z. 74 (1960), 29-38.

4. - Invariants for modules over commutative separable orders, Quart. J. Math. Oxford Ser. 16 (1965), 193-232.

5. H. B. Mann, On integral bases, Proc. Amer. Math. Soc. 9 (1958), 167-172.

6. - Introduction to algebraic number theory, The Ohio State University Press, Columbus, 1955.

7. L. R. McCulloh, Integral bases in Kummer extensions of Dedekind fields, Canad. J. Math. 15 (1963), 755-765.

8. E. Weiss, Algebraic number theory, McGraw-Hill, New York, 1963.

UNIVERSITY OF ILLINOIS 